\title{
Cuadro de mando integral en la toma de decisiones de las cooperativas agrarias de la región Junín
}

\section{Comprehensive scorecard in the decision making of the agricultural cooperatives of the Junín region}

\author{
César Pariona C.(1) y Hugo Peña H. ${ }^{(1)}$ \\ (1) Facultad de Contabilidad, Universidad Nacional del Centro del Perú, Perú \\ Email: cpariona@uncp.edu.pey hcpenia@uncp.edu.pe
}

\section{RESUMEN}

El presente trabajo de investigación, identifica que gran parte de las cooperativas y sus directivos, tienen la falta de cultura de control; por cuanto, no se encuentran predispuestos en aceptar los recientes modelos administrativos. Esta situación es consecuencia de la inadecuada utilización de las herramientas de administración, conllevando por esta razón; a formular la siguiente pregunta ¿Qué modelo de control gerencial permite el fortalecimiento en la toma de decisiones de las cooperativas agrarias de la región Junín?

Ante esta situación, como respuesta a la problemática planteada, se propone la siguiente hipótesis: El modelo de control gerencial basado en el cuadro de mando integral permite el fortalecimiento en la toma de decisiones de las cooperativas agrarias de la región Junín.

Para el efecto, el presente trabajo, se ha orientado al cumplimiento del siguiente objetivo: Proponer el modelo de control gerencial que permite el fortalecimiento en la toma de decisiones de las cooperativas agrarias de la región Junín

En la metodología, se explica que la investigación es del tipo aplicada del nivel descriptivo; asimismo, los métodos de investigación utilizados son: la observación, el descriptivo e inductivo. Las técnicas de investigación estuvieron relacionados en la recopilación, tabulación, tratamiento y análisis de datos. La población estuvo conformada por trabajadores de las veinte cooperativas agrarias de la región Junín.

Palabras Claves: Mando integral - Toma de decisiones - Cooperativa agraria - Control gerencial

\section{ABSTRACT}

The present work "Comprehensive scorecard in decision making in the agricultural cooperatives of the Junín Region, began with the identification that a large part of these companies and their managers have the lack of control culture, affecting traditional processes that are not competitive By rejection to the new administrative tendencies, this situation is consequence of the inadequate use of the tools of administration, implying for this reason; To ask the following question What model of managerial control allows the strengthening of decision-making in the agricultural cooperatives of the Junín Region?

In this situation; In response to the problem raised, the following hypothesis is proposed. The management control model based on the integral scorecard allows the strengthening of decision-making in agricultural cooperatives.

For the purpose; The present work has been oriented to the fulfillment of the following objective. To formulate the managerial control model that allows the strengthening of decision-making in the agrarian cooperatives of the Junín Region

In the methodology part it is explained that the present thesis, the type of investigation is basic, the level of investigation is descriptive - explanatory, likewise; The research methods used are observation, analysis and synthesis, descriptive - explanatory. The research techniques were related in the collection, tabulation, treatment and analysis of data. The population was made up of workers from the 20 agrarian cooperatives of the Junín Region

Keywords: Integral scorecard - Decision making - Agricultural cooperatives - Management control 


\section{INTRODUCCIÓN}

El presente estudio, está orientado a proponer el modelo de control gerencial que permita el fortalecimiento en la toma de decisiones de las cooperativas agrarias de la región Junín, con la finalidad que los investigadores orienten estudios del tipo aplicativo con la construcción de herramientas administrativas que les permitan alcanzar eficacia de sus operaciones.

Si las cooperativas de la región Junín, aún no logran la eficacia en sus operaciones, es porque no son capaces de lograr competitividad; por ello, se busca en el cuadro de mando integral lineamientos a que estas cooperativas alcancen efectividad empresarial, aprovechando su especialidad de dedicarse a la actividad agropecuaria desde hace 55 años.

Para llegar a solucionar la problemática, el trabajo se ha desarrollado en tres temas.

Tema No 01, relacionado al estudio de las cooperativas agrarias que funcionan dentro de la región Junín.

El tema No 02, está relacionado al estudio cuadro de mando integral, señalando pautas para su aplicación.

El tema No 03, está relacionado al estudio de la toma de decisiones, señalando pautas para su aplicación.

\section{MATERIALES Y MÉTODOS}

\section{Materiales}

El trabajo de investigación se realizó en las cooperativas agrarias de la región Junín; para lo cual, los principales materiales que se emplearon fueron: útiles de escritorio, separatas relacionadas con el estudio, bibliografía adecuada al tema de investigación, equipos de oficina, las encuestas y computadora.

\section{Método}

El método empleado fue desde el planteamiento de carácter específico a resultados generales. El presente trabajo es una investigación aplicada, al depender de sus descubrimientos y aportes teóricos; por ello, busca confrontar la teoría con la realidad. Es el estudio y aplicación de problemas concretos en circunstancias y características concretas. Esta forma de investigación se dirige a su aplicación inmediata y el desarrollo de teorías (Valderrama, 2007).

La investigación es del nivel descriptivo, al respecto Bernal (2016), señala que la investigación descriptiva es uno de los tipos o procedimientos investigativos más popular y utilizado por los principiantes en la actividad investigativa. Los trabajos en los pregrado y en muchas maestrías, son de carácter eminentemente descriptivos. En tales investigaciones se muestran, narran, reseñan o identifican hechos, situaciones, rasgos y características de un objeto de estudio; se realizan diagnósticos, perfiles o se diseñan productos, modelos, prototipos, guías, etc.; pero no se dan explicaciones o razones de las situaciones, los hechos, los fenómenos y otros.

\section{Diseño}

El diseño para el desarrollo del presente trabajo de investigación fue el transversal, no experimental, descriptivo, conforme al esquema siguiente:

$M=$ Muestra (trabajadores encuestados)

$O_{X}=$ Cuadro de mando integral

$O y=$ Toma de decisiones

\section{Técnicas utilizadas}

- Entrevista

- Encuestas (cuestionario)

- Observación

\section{Instrumentos}

Los instrumentos de investigación utilizados son:

- Guía de entrevista. Dentro de la guía de entrevista se elaboró la entrevista estructurada con preguntas libres.

- Cuestionario. El cuestionario que se aplicó, son de preguntas cerradas y preguntas abiertas.

- Guía de observación. Se utilizó la observación directa.

\section{Fuentes}

La fuente de información primaria fueron las siguientes:

- Observación por medio de informes.

- Entrevistas con la gerencia, contador o propietario.

\section{Técnicas de procesamiento y análisis de datos} recolectados

Se realizaron en función a las variables establecidas.

Se utilizó el procesador sistematizado computarizado. El procesamiento de datos, se realizó con Excel.

\section{RESULTADOS}

\section{Resultados de los instrumentos de investigación}

Se realizó un trabajo de investigación sobre la propuesta del cuadro de mando integral para la toma de decisiones de las cooperativas agrarias de la región Junín. Para la recopilación de opiniones de los factores que afectaban al desarrollo de las mismas, se concluyó que las cooperativas agrarias de la región Junín no utilizan, como herramienta de gestión para la toma de decisiones, el cuadro de mando integral; motivo por el cual, se adjunta, al presente, una propuesta del cuadro de mando integral aplicable para este tipo de entidades.

\section{DISCUSIÓN}

La investigación realizada sobre el cuadro de mando integral en la toma de decisiones en las cooperativas agra- 
rias de la región Junín, tuvo como finalidad el fortalecimiento de la toma de decisiones programadas de las cooperativas agrarias de la región Junín, teniendo como referencia al resultado de su desempeño establecido dentro del cuadro de mando integral.

Para ello, citamos a Dávila (1999), quién señala que el "cuadro de mando integral es una herramienta útil para dirigir empresas de forma proactiva en el corto y en el largo plazo. Su eficacia radica en una buena comprensión de sus fundamentos, una aplicación completa que implique a la dirección de la compañía”.

También, dentro del control interno y el valor agregado, Mantilla (2009), señala que los "controles de alto valor agregado son el autocontrol, el empoderamiento y el liderazgo; esto es gente capaz, con poder, íntegra e informada. Que trabajan en un contexto de planeación estratégica y mejoramiento continuo...”.

En ese mismo orden de ideas, Villajuana (2001), referente a la implementación del tablero de gestión estratégica, señala que el "Balanced Scoredcard" es una herramienta clave para medir la eficacia y eficiencia de la gestión de toda organización. Su mayor valor reside en permitir medir la generación de valor de los intangibles y en que utiliza los demás sistemas convencionales de forma complementaria para obtener mejores resultados.

A su vez Chiavenato, dentro de los elementos del proceso administrativo, expresa que la "planeación define lo que pretende realizar la organización en el futuro y cómo debe realizarlo. Por esta razón, la planeación es la primera función administrativa, y se encarga de definir los objetivos para el futuro desempeño organizacional y decide sobre los recursos y tareas necesarias para alcanzarlos de manera adecuada. Gracias a la planeación, el administrador se guía por los objetivos buscados y las acciones necesarias para conseguirlos, basados en algún método, plan o lógica, en vez de dejar todo al azar. La planeación produce planes basados en objetivos y en los mejores procedimientos para alcanzarlos de manera apropiada, Por tanto, planear incluye la solución de problemas y la toma de decisiones en cuanto alternativas para el futuro".

Asimismo, trabajos de investigación relacionados al tema, muestran conclusiones, como la de Miculicich (2011), quien en su trabajo de investigación titulado "Sistema de gestión de estrategias e indicadores utilizando metodologías de inteligencia de negocios en una universidad privada", concluye que el "soporte al diseño de los CMI y CM es lo suficientemente interactivo para permitir al usuario realizar su labor fácilmente y la información mostrada es lo suficientemente clara y confiable a los usuarios...”.

Finalmente tenemos a Marín (2010), quien en su trabajo de investigación titulado "Desarrollo de una herramienta de soporte para el cuadro de mando integral”, ma- nifiesta en su conclusión que "La teoría del cuadro de mando integral es una metodología eficiente y eficaz para la administración de la estrategia de las empresas, utilizarla adecuadamente permite medir las operaciones realizadas por la organización y tomar las acciones necesarias para el logro de los objetivos a alcanzar. La herramienta del cuadro de mando integral permite visualizar, en una única pantalla, la situación actual de la organización mediante la presentación ordenada y agrupada de las perspectivas, objetivos, iniciativas, indicadores y metas definidas para la administración de la estrategia adoptada".

Siendo las cooperativas agrarias de la región Junín, empresas referentes de la reforma agraria en el Perú (D.L $\mathrm{N}^{\circ} 17716$ y D.S 240-69), éstas no han tenido la oportunidad de llevar a cabo gestiones acompañado de herramientas administrativas que midan la eficacia administrativa, bajo los indicadores financieros y no financieros que sustenta el cuadro de mando integral.

\section{CONCLUSIONES}

Las cooperativas agrarias de la región Junín, a través de los directivos, ejecutivos y trabajadores, tienen la falta de cultura de control; por cuanto, no se encuentran predispuestos en aceptar los recientes modelos administrativos, pese a tener exclusividad en la actividad pecuaria en gran escala, tales como vacunos, ovinos, camélidos sudamericanos y piscigranja y; en la actividad del agro, manejan el cultivo de café, cacao, entre otros. Por ello, no cuentan con herramientas de gestión que les permita medir su desempeño en términos financieros y no financieros, para que puedan tomar decisiones que les permita alcanzar sus objetivos empresariales.

\section{REFERENCIAS BIBLIOGRÁFICAS}

Bellman, R. (1964): Teoría de control en Scientific American, pág 186

Bernal, C. Metodología de la investigación científica, cuarta edición, editorial PEARSON, Colombia 2016

Mantilla, S. Auditoria de control interno, 2da edición, Bogotá, Ecoe Ediciones 2009

Pino, R. (2006) Metodología de la investigación, segunda edición, Lima - Perú

Poch, R. (2000) Los circuitos informáticos en la administración empresarial, ediciones Gestió 2000 -Barcelona

Terry, G. (1984) Principios de administración, México, editorial CECSA

Valderrama, S. Pasos para elaborar proyectos y tesis de investigación científica, editorial San Marcos EIRL, primera edición 2007 Lima - Perú 


\section{Tesis}

Iculicich, L. (2011) Tesis titulada "Sistema de gestión de estrategias e indicadores utilizando metodologías de inteligencia de negocios en una universidad privada, para optar por elTítulo de Ingeniero Informático en la Universidad Pontificia Católica del Perú

Marín, R. (2010) Tésis titulada "Desarrollo de una herramienta de soporte para el cuadro de mando integral, para optar por el Título de Ingeniero Informático en la Universidad Pontificia Católica del Perú

\section{Dispositivos legales}

El peruano: Decreto Ley N ${ }^{\circ}$. 17716: Ley de Reforma Agraria, Publicado el 24 de junio del año 1969

El Peruano: Decreto Supremo N 240-69: Reglamento de cooperativas agrarias y comunales: contiene las funciones y organización de las cooperativas agrarias

El Peruano: Decreto Legislativo $\mathrm{N}^{\circ} 085$ Ley general de cooperativas, publicada el 20 de mayo de 1981

\section{Internet}

Chiavenato, I. Administración en los nuevos tiempos. Consultado el 27 de diciembre de 2016 de http: / / cvonline.uaeh.edu.mx/Cursos/Maestria/MTE/ Gen02/admon_gest_ntics/unidad_1/U1_Nydamonactual.pdf

Dávila, A. (1999): Nuevas herramientas de control: El cuadro de mando Integral. Consultado el 05 de enero de 2017 de http: / / http: / / campus.easp.es / recursos/DiplomaGestion/documentos/5-afondo.pdf

Villajuana, P. (2001): Como implementar el tablero de gestión estratégica, consultado el 20 de diciembre de 2016 de http://sisbib.unmsm.edu.pe/bibvirtualdata/publicaciones/estudios_ciencias/v1n1/ a14_p247.pdf 


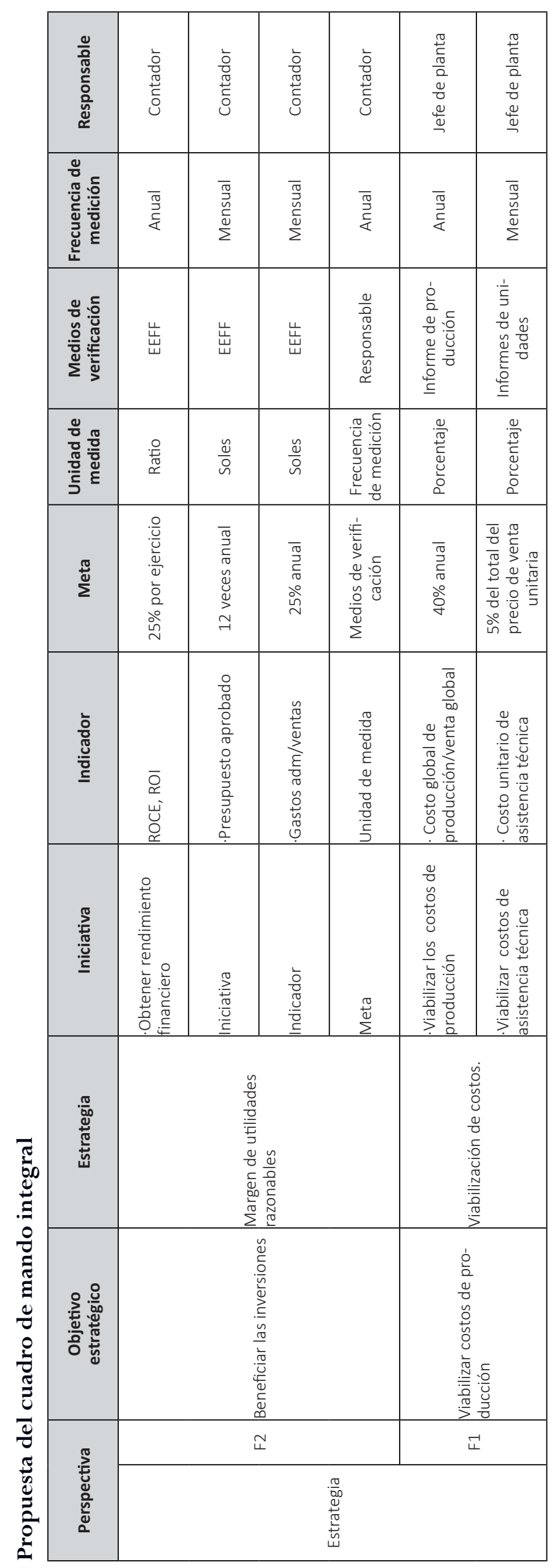

\begin{tabular}{|c|c|c|c|}
\hline 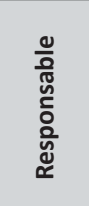 & 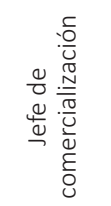 & $\begin{array}{l}\frac{0}{0} \\
\frac{0}{0} \\
\frac{0}{0} \\
\frac{0}{0} \\
\frac{\Phi}{9}\end{array}$ & 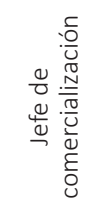 \\
\hline 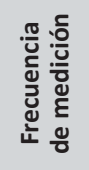 & 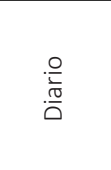 & 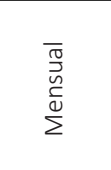 & 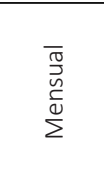 \\
\hline 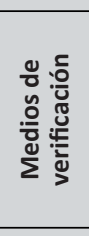 & 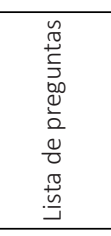 & 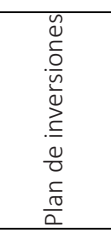 & 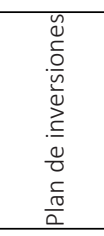 \\
\hline 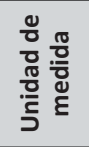 & 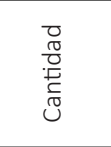 & 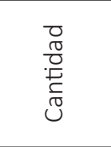 & $\frac{\ddot{d}}{\dot{\omega}}$ \\
\hline 茄 & 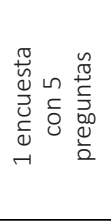 & 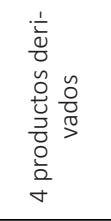 & 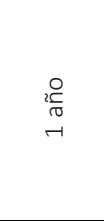 \\
\hline 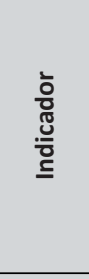 & 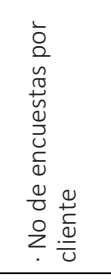 & 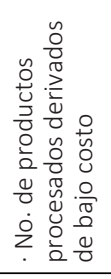 & 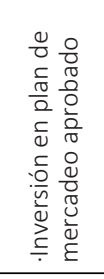 \\
\hline 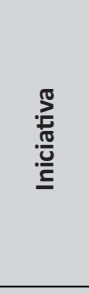 & 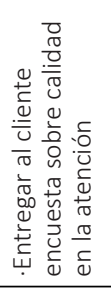 & 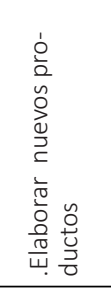 & 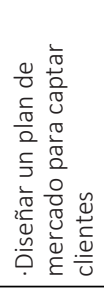 \\
\hline 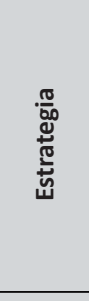 & 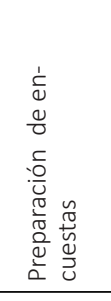 & 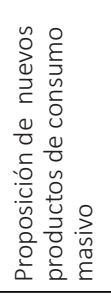 & 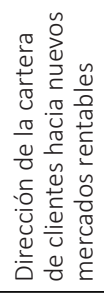 \\
\hline 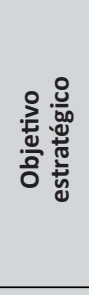 & 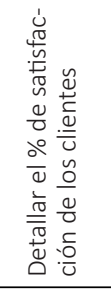 & 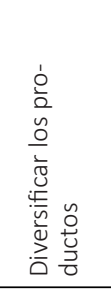 & 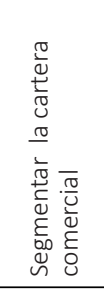 \\
\hline \multirow{2}{*}{ 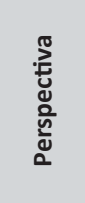 } & 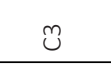 & ช & $\vec{U}$ \\
\hline & \multicolumn{3}{|c|}{ 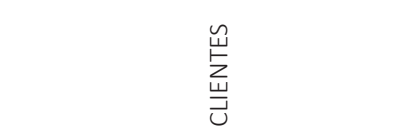 } \\
\hline
\end{tabular}




\begin{tabular}{|c|c|c|c|c|c|}
\hline 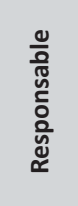 & 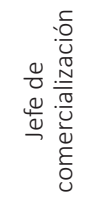 & 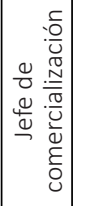 & 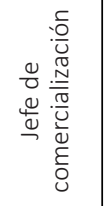 & 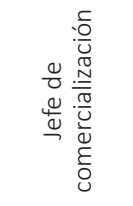 & 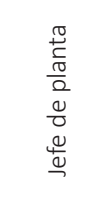 \\
\hline 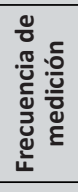 & 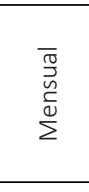 & 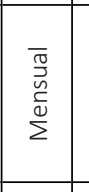 & 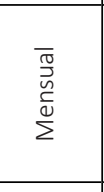 & 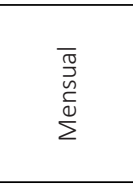 & 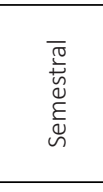 \\
\hline 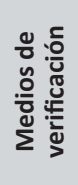 & 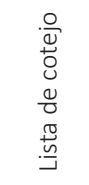 & 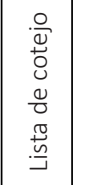 & 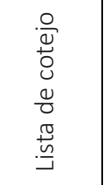 & 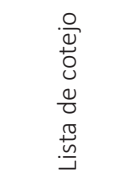 & 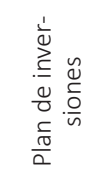 \\
\hline 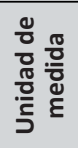 & 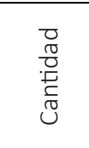 & 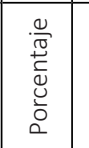 & 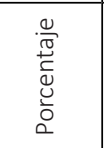 & 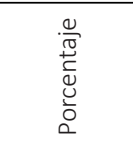 & 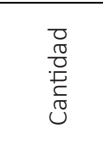 \\
\hline$\stackrel{\mathbb{g}}{\Sigma}$ & 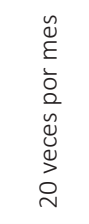 & ઠे & ذे & ஃें & 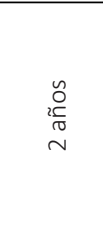 \\
\hline 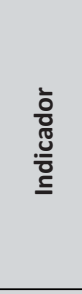 & 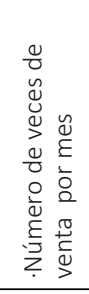 & 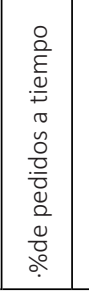 & 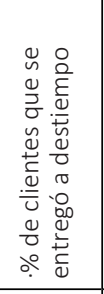 & 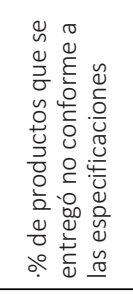 & 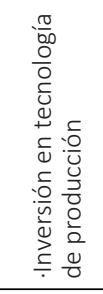 \\
\hline 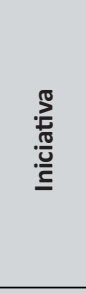 & & 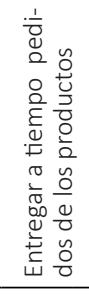 & & 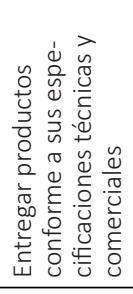 & 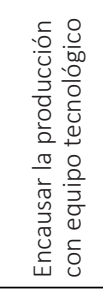 \\
\hline 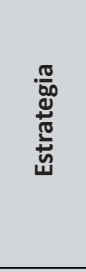 & & & 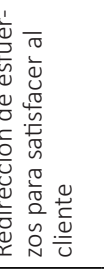 & & 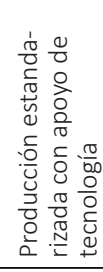 \\
\hline 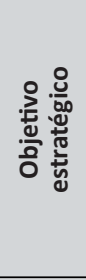 & & & 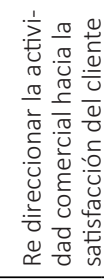 & & 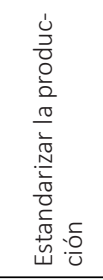 \\
\hline \multirow{2}{*}{ 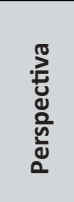 } & & & $\Sigma$ & & $\vec{a}$ \\
\hline & \multicolumn{5}{|c|}{ 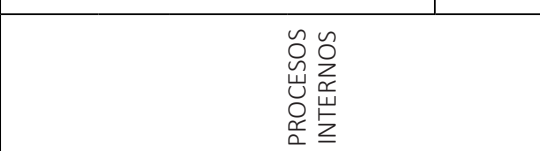 } \\
\hline
\end{tabular}

\begin{tabular}{|c|c|c|c|c|c|}
\hline 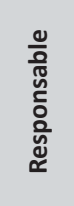 & 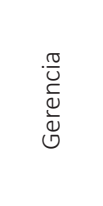 & 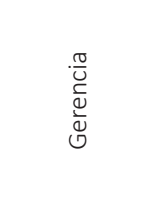 & 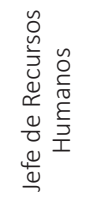 & 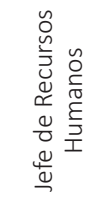 & 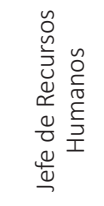 \\
\hline 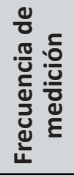 & $\begin{array}{l}\overline{\widetilde{J}} \\
\overline{\bar{N}} \\
\tilde{\sigma}^{\frac{\pi}{2}}\end{array}$ & 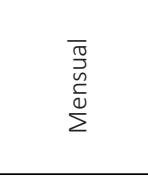 & 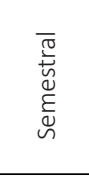 & 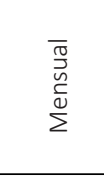 & 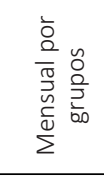 \\
\hline 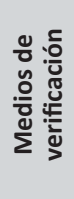 & 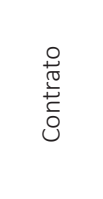 & $\begin{array}{l}\frac{0}{0} \\
\frac{0}{0} \\
\frac{0}{0} \\
\varepsilon_{0}^{0} \\
\sum\end{array}$ & 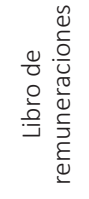 & 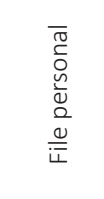 & 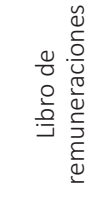 \\
\hline 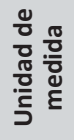 & 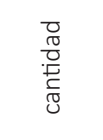 & 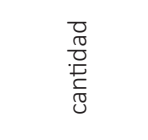 & 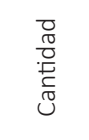 & 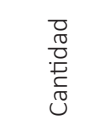 & 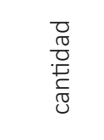 \\
\hline$\sum^{\frac{\pi}{0}}$ & 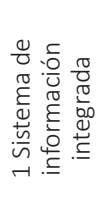 & 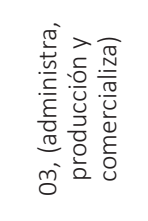 & 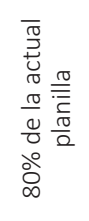 & 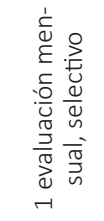 & 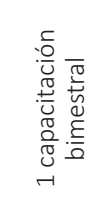 \\
\hline 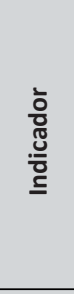 & 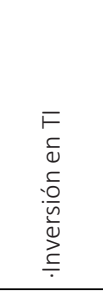 & 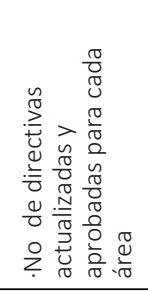 & 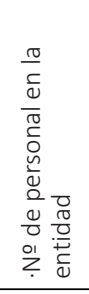 & 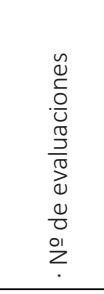 & 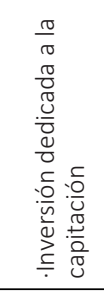 \\
\hline 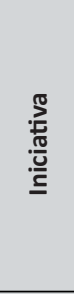 & 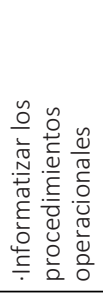 & 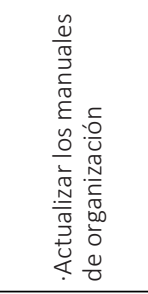 & 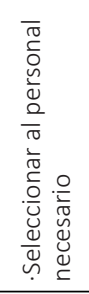 & 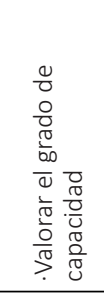 & 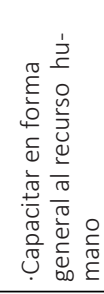 \\
\hline 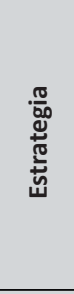 & 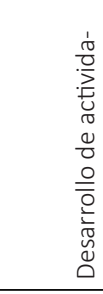 & 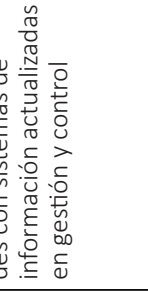 & & 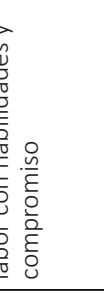 & 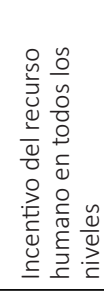 \\
\hline 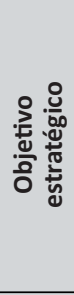 & & 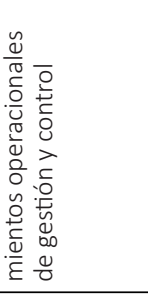 & & & 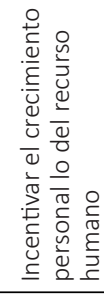 \\
\hline \multirow{2}{*}{ 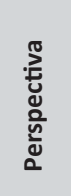 } & & q & & y & $\overrightarrow{<}$ \\
\hline & \multicolumn{5}{|c|}{ 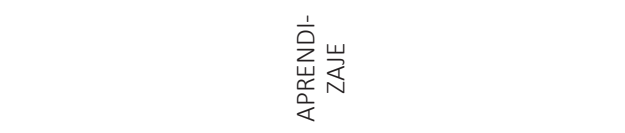 } \\
\hline
\end{tabular}

